\title{
Malaria: revisión retrospectiva de 12 casos no autóctonos en Chile
}

\author{
Carlos Pérez $C^{1,4}$, René Baudrand $B^{1,4}$, Jaime Labarca $L^{1,4}$, \\ Cecilia Perret $P^{2,4}$, Max Andresen $H^{1,4}$, Ana Mạ Guzmán $D^{3,4}$. \\ Malaria: Report of 12 \\ non autochthonous cases
}

\begin{abstract}
Malaria is a protozoan infection caused by four Plasmodia species transmitted by female Anopheles mosquito. Nearly $40 \%$ of the world population is at risk of acquiring the disease because of increasing resistance to treatment, climate changes and travels to endemic zones. We report twelve patients with diagnosis of malaria, supported by the identification of parasites on blood smear. All cases had traveled to endemic zones (Peru, Ecuador, Central America, Africa), but only three used chemoprophylaxis. Seven cases were infected with Plasmodium vivax and five cases with P. falciparum. Three of latter required intensive care. All patients were treated with standard drugs according to the severity and Plasmodium specie, with excellent results and no mortality (Rev Méd Chile 2006; 134: 421-5).

(Key words: Malaria; Plasmodium falciparum; Plasmodium vivax)
\end{abstract}

Recibido el 27 de octubre, 2004. Aceptado el 6 de diciembre, 2005.

${ }^{1}$ Departamento de Medicina Interna, ${ }^{2}$ Departamento de Pediatría, ${ }^{3}$ Unidad Docente Asociada Laboratorio Clínico, ${ }^{4}$ Programa de Enfermedades Infecciosas, Facultad de Medicina, Pontificia Universidad Católica de Chile. Santiago de Chile.

L a malaria es una infección protozooaria sistémica producida por cuatro especies de Plasmodium: falciparum, vivax, ovale y malarie. Es transmitida por el mosquito Anopheles hembra, aunque también está descrita la transmisión materno-fetal, por transfusiones, inoculación accidental o trasplante de órganos, siendo el hombre el único reservorio de importancia clínica ${ }^{1}$. Considerada una de las plagas más antiguas de la humanidad, produce cada año 300 a 500 millones de nuevos casos y 3 millones de muertes, lo que genera grandes costos económicos y afecta el desarrollo de países donde la malaria es endémi-

Correspondencia a: Dr. Carlos Pérez Cortes. Lira 44, Santiago, Chile. E mail: cape@med.puc.cl $\mathrm{ca}^{2}$. Aproximadamente, $40 \%$ de la población mundial se encuentra en riesgo de adquirir esta enfermedad, debido a la creciente resistencia al tratamiento e insecticidas, cambios climáticosecológicos y a un aumento importante de viajes a zonas endémicas ${ }^{3}$.

En Chile se logró erradicar la malaria existente en la provincia de Tarapacá desde 1913, con la campaña a cargo del profesor Juan Noé, entre los años 1937 y 19474,5. Desde entonces, sólo se han reportado casos aislados y una serie clínica de 9 casos no autóctonos entre los años 1972 y 1984, en los cuales existió el antecedente epidemiológico de permanencia en zonas maláricas ${ }^{6}$. Según datos del Departamento de Epidemiología del Ministerio de Salud, en los últimos años se ha producido un aumento de pacientes infectados, 
registrándose entre 1990 y 2001, 66 nuevos casos en los distintos Servicios del país y 5 fallecimientos ${ }^{7}$. Según el boletín de enfermedades de notificación obligatoria ministerial, en 2003 hubo 6 nuevos casos y en 2004 se presentaron 7 nuevos casos.

El aumento del turismo en los últimos años ha incrementado el número de chilenos y extranjeros expuestos a malaria. Consideramos oportuna la revisión de los casos tratados en nuestro hospital para dar a conocer nuestra experiencia en el diagnóstico y manejo de esta patología.

\section{Pacientes y MÉTodos}

Se revisaron retrospectivamente las fichas clínicas de 12 pacientes hospitalizados en el Hospital Clínico de la Universidad Católica de Chile entre 1994 y 2003. En todos los casos se confirmó malaria por identificación de los parásitos en la sangre, por tecnólogos médicos o médicos con experiencia en la interpretación de este tipo de frotis.

Se analizaron sus datos, registrándose: edad, sexo y nacionalidad; antecedentes de estadía en zona malárica, antecedente de malaria previa y utilización de quimioprofilaxis, insecticidas o mosquitero; diagnóstico de ingreso, demora en realizar el diagnóstico y descripción de los síntomas presentados. Se registraron la duración de la hospitalización, la evolución y complicaciones, además de exámenes de laboratorio generales e identificación del tipo de Plasmodium y el tratamiento aplicado.

\section{Resultados}

La muestra incluyó a 7 hombres y 5 mujeres. Las edades fluctuaron desde 18 a 64 años (promedio $=32,3$ años). Diez casos fueron pacientes chilenos y dos casos extranjeros, ambos residentes en África, que estaban de paso por el país.

Antecedentes epidemiológicos. Todos los casos descritos tenían el antecedente de permanencia en zonas endémicas maláricas: Ecuador (2 casos), selva amazónica Perú-Ecuador (2 casos), varios países en África (2 casos), Nicaragua-Panamá-
Costa Rica (1 caso), República Dominicana (1 caso), Nueva Guinea (1 caso), Costa de Marfil (1 caso), Mozambique (1 caso) e India (1 caso). Esta estadía se originó por turismo (8 casos), trabajo (2 casos) y por residencia en dicha zona (2 casos: 1 chileno y 1 extranjero). De los 10 pacientes chilenos que viajaron a zonas endémicas de malaria, sólo 3 recibieron quimio-profilaxis: 2 con cloroquina y 1 con mefloquina. Cabe destacar que en 3 casos existía el antecedente de malaria previa (1 chileno y 2 extranjeros).

Cuadro clínico. Todos los pacientes tuvieron un síndrome febril cuya duración osciló entre 7-21 días antes de consultar, con un rango de temperatura entre $37,5^{\circ} \mathrm{C}$ y $40^{\circ} \mathrm{C}$. La mayoría de los pacientes presentaron el cuadro clásico de fiebre, calofríos y mialgias, además de otras manifestaciones que se resumen en la Tabla 1.

En general, el diagnóstico de ingreso correspondió a "síndrome febril en estudio», con la excepción de dos casos en los que se formularon como diagnósticos: pielonefritis aguda en una paciente con un embarazo de 28 semanas y fiebre tifoidea en otra paciente. En 8 pacientes se planteó malaria como opción diagnóstica al ingreso. El número de días hospitalizados promedio fue de 7,6 días con un rango entre 2 y 30 días.

Evolución y complicaciones. Seis pacientes evolucionaron sin complicaciones, pero 9 requirieron transfusión de glóbulos rojos; 3 presentaron trom-

Tabla 1. Cuadro clínico en 12 casos de malaria

\begin{tabular}{|lcr|}
\hline Síntomas & Número & $(\%)$ \\
\hline Fiebre & 12 & $(100)$ \\
Calofríos & 12 & $(100)$ \\
Mialgias & 7 & $(58)$ \\
Náuseas - vómitos & 3 & $(25)$ \\
Cefalea & 3 & $(25)$ \\
Compromiso de conciencia & 1 & $(8)$ \\
& & \\
Signos & Número & $(\%)$ \\
\hline Esplenomegalia & 7 & $(58)$ \\
Hepatomegalia & 6 & $(50)$ \\
Ictericia & 4 & $(33)$ \\
\hline
\end{tabular}


Tabla 2. Casos que presentaron complicaciones (corresponden a Plasmodium Falciparum)

\begin{tabular}{|lccl|}
\hline Sexo & $\begin{array}{c}\text { Edad } \\
\text { (años) }\end{array}$ & $\begin{array}{c}\text { № días } \\
\text { hospitalización }\end{array}$ & Complicaciones \\
\hline $\begin{array}{l}\text { Femenino } \\
\text { Masculino }\end{array}$ & 26 & 8 & $\begin{array}{l}\text { Sepsis + insuficiencia respiratoria + hepatitis } \\
\text { Masculino }\end{array}$ \\
37 & 13 & $\begin{array}{l}\text { Shock séptico + coagulación intravascular } \\
\text { diseminada }\end{array}$ \\
& 35 & 30 & $\begin{array}{l}\text { Shock séptico + distrés respiratorio + hepatitis } \\
\text { + pancreatitis + insuficiencia renal aguda } \\
\text { (hemodiálisis) }\end{array}$ \\
\hline
\end{tabular}

bocitopenia y requirieron terapia transfusional con plaquetas y 3 fueron ingresados a unidades de cuidados intensivos. Estos últimos tres casos se detallan en la Tabla 2 y tienen la particularidad de corresponder todos a Plasmodium falciparum.

Exámenes de laboratorio (Tabla 3). De los exámenes generales el más útil fue el hemograma, por cuanto permitió realizar el diagnóstico, caracterizar el tipo de Plasmodium y ayudó en el diagnóstico diferencial de las anemias hemolíticas. En esta serie no fue necesario realizar examen de gota gruesa para el diagnóstico. Los valores alterados de función renal y hepática correspondieron a los casos que presentaron complicaciones. El sedimento de orina alterado (cilindruria y hematuria) se observó en una paciente cursando un embarazo, con

Tabla 3. Exámenes de laboratorio en 12 casos de malaria

\begin{tabular}{|lcc|}
\hline Laboratorio & Número & $(\%)$ \\
\hline Anemia & 10 & $(83)$ \\
Trombocitopenia & 8 & $(67)$ \\
Hiperbilirrubinemia & 6 & $(58)$ \\
Leucopenia & 3 & $(25)$ \\
Frotis positivo para: & & \\
$\quad$ P vivax & 7 & $(58)$ \\
P falciparum & 5 & $(42)$ \\
\hline
\end{tabular}

urocultivo negativo, y en un paciente que desarroló una insuficiencia renal aguda.

Con respecto al tipo de Plasmodium, registramos 7 casos de P. vivax y 5 casos de P. falciparum. Esta identificación se realizó con el frotis del hemograma.

Tratamiento. En los casos de malaria por P. vivax se utilizó la asociación de cloroquina (dosis $0 \mathrm{~h}=1$ g; dosis $6 \mathrm{~h}=500 \mathrm{mg}$; dosis $24 \mathrm{~h}=500 \mathrm{mg}$ y dosis $48 \mathrm{~h}=500 \mathrm{mg}$ ), más primaquina (15 mg base por 14 días).

Los casos de malaria por P. falciparum fueron tratados con el esquema asociado de quinina (600 $\mathrm{mg} \mathrm{c} / 8 \mathrm{~h})$ y doxiciclina $(100 \mathrm{mg} \mathrm{c} / 12 \mathrm{~h})$ por 7 días. En el caso más severo por P. falciparum, dada la gravedad del cuadro clínico y dificultad de uso de vía oral, se realizó un tratamiento con quinidina endovenosa (gluconato de quinidina) en infusión continua (10 $\mathrm{mg} / \mathrm{kg}$ por 1 hora, luego $0,02 \mathrm{mg} / \mathrm{kg} / \mathrm{min}$ ). No hubo mortalidad atribuible a malaria.

\section{Discusión}

Desde 1945 no existen reportes de casos de malaria autóctona en el país, fruto de una campaña de erradicación en la provincia de Tarapacá ${ }^{8}$. En algunos valles de la primera región se han encontrado Anopheles pseudopunctipenis, pero no se encuentran infectados por el parásito de la malaria. Sin embargo, a nivel mundial esta enfer- 
medad constituye una de las patologías infecciosas más prevalentes, especialmente en zonas endémicas.

Actualmente, consecuencia de la globalización $\mathrm{y}$ al fomento del turismo, existen viajes hacia y desde zonas maláricas, lo que explica el aumento en los casos observados en los últimos años.

Casi $90 \%$ de los casos mundiales de malaria corresponden a P. falciparum y ocurren en territorio africano, en los cuales se describen las mayores tasas de mortalidad, que alcanzan hasta 25\% de los casos de malaria severa. En Asia, Oceanía, Centroamérica y Sudamérica existe endemia de P. vivax (asociado a mejor pronóstico) y P. falciparum, sumado a un mayor acceso a diagnóstico y tratamiento, por lo que las cifras de mortalidad son mejores ${ }^{9}$.

Los datos ministeriales chilenos revelan que se han reportado más casos en la última década, con una tasa de 6,6 casos/año en el período 19972001, cifra que se ha mantenido relativamente constante, siendo las notificaciones generalmente de Servicios de Salud de la Región Metropolitana. De la serie reportada por Schenone y cols. sólo $30 \%$ de los 66 pacientes eran de nacionalidad chilena, todos habían estado en zonas maláricas (principalmente en África y Sudamérica), hubo una mortalidad de $8,8 \%$ y no se identificaron los subtipos de Plasmodium?

Una serie del Hospital Lucio Córdova, cita 9 casos de malaria no autóctona en 12 años de observación, todos correspondientes a P. vivax ${ }^{6}$. En nuestra serie, describimos 12 casos en 9 años, lo que corrobora que es una entidad poco frecuente y en ascenso. Existen semejanzas entre ambas series, por cuanto la totalidad de los pacientes tiene el antecedente de estadía en zonas maláricas y el hecho de debutar como síndromes febriles. Al igual que en nuestra serie, se plantearon como diagnósticos alternativos de ingreso fiebre tifoidea e infección urinaria; sin embargo el antecedente epidemiológico y el examen del frotis del hemograma permitieron realizar el diagnóstico de malaria.
Con respecto a las manifestaciones clínicas, son similares a las descritas en series anteriores, excepto por presentar casos más complejos, que requirieron un manejo multidisciplinario en unidades de paciente crítico. Estas complicaciones tenían relación con el aparato biliar (ictericia y dolor en el hipocondrio derecho), el sistema nervioso central (compromiso de conciencia), el estado hemodinámico (shock) y el deterioro de la función renal (1 caso requirió hemodiálisis de urgencia). Esto podría explicarse por la mayor proporción de casos por P. falciparum en esta serie clínica.

El tratamiento realizado estuvo de acuerdo a lo descrito en la literatura ${ }^{10}$, teniendo buen resultado, inclusive en los pacientes más complicados, sin mortalidad asociada. En general, en casos severos causados por P. falciparum se prefiere usar tratamiento endovenoso con quinidina 0 quinina, utilizando los derivados de artemisinina para casos con resistencia a quinina ${ }^{11}$. En este grupo de pacientes se requiere de procedimientos invasivos, estudio con imágenes, asistencia nutricional, soporte y monitorización en unidades de cuidado intensivo, con apoyo de distintas especialidades médicas.

Observamos que pese al mayor desarrollo del turismo a nivel mundial, sólo una minoría de los pacientes que viajan a zonas maláricas recibe quimioprofilaxis, con el consiguiente mayor riesgo de infectarse por algún Plasmodium en regiones endémicas. Llama la atención que, pese a la mayor información disponible y el desarrollo de la medicina del viajero, los chilenos que viajan a zonas endémicas de malaria no toman profilaxis ni se informan de los riesgos de otras enfermedades transmisibles.

En resumen, se debe tener una alta sospecha frente a síndromes febriles en pacientes con antecedentes de viajes a zonas maláricas en los últimos meses, considerando que el período de incubación es variable, y mediante un hemograma, examen ampliamente disponible, orientarse hacia un correcto diagnóstico y tratamiento. 


\section{REFERENCIAS}

1. Stanley J. Malaria. Emerg Med Clin North Am 1997; 15: 113-55.

2. OLiaro P, Cattani J, Wirth D. Malaria, the submerged disease. JAMA 1996; 275: 230-3.

3. World Health Organization. The World Health Report 1997: Conquering suffering, en riching humanity. Geneva: WHO Office of Information, 1997.

4. Neghme A. La erradicación de la Malaria, un desafío al mundo. Rev Méd Chile 1960; 88: 24750.

5. Gutiérrez Fu, Neghme A, Albi De la Fuente H. Estado actual del control del anofelismo en Chile. Rev Chil Hig Med Prev 1950; 12: 67-71.
6. Ramírez G, Hazbún M, Palomino C. Características clínicas de 9 casos de Malaria no autóctona. Rev Méd Chile 1988; 116: 143-7.

7. Schenone H, Olea A, Rojas A, García N. Malaria en Chile: 1913-2001. Rev Méd Chile 2002; 130: 11706.

8. Noe J, Bertín V, Gutiérrez J, Neghme A. Diez años de lucha antimalárica en Chile. Riv Parassitol 1949; 10: 5-24.

9. Suh K, Kain K, Keystone J. Malaria. CMAJ 2004; 170: 1693-702.

10. White NJ. The treatment of Malaria. N Engl J Med 1996; 335: 800-6.

11. Trampuz A, Jereb M, Muzlovic I, Prabhu RM. Clinical review: Severe malaria. Crit Care 2003; 7: 315-23. 